\title{
Für eine zukunftsfähige und umweltorientierte Finanzpolitik
}

\begin{abstract}
Die Vereinigung für ökologische Wirtschaftsforschung (VÖW) e.V. begrüßt in einem Miłte Oktober veröffentlichten Diskussionspapier, den Einstieg in die Ökologische Steuerreform. Sie stellt ein zentrales Element auf dem Weg zu einer nachhaltigen Gesellschaft dar. Bei allem notwendigen Pragmatismus zur Umsetzung dieser Vision sieht die VöW jedoch bei der Ausgestaltung der anvisierten weiteren Stufen dringenden Reformbedarf. Dieser wird ausgehend von den Kriterien Langfristigkeit, ökologische Wirksamkeit, soziale Ausgewogenheit und psychologische Schlüsselwirkung abgeleitet. Mittelfristig ist eine breiłere Ökologiesierung der Finanzpolitik in Angriff zu nehmen.
\end{abstract}

(...) Seit dem 1. April 1999 hat Deutschland den Einstieg in die Ökologische Steuerreform vollzogen. (...) Dies ist nicht zuletzt auch ein Erfolg der ökologischen Wirtschaftsforschung der letzten 20 Jahre und wird von der vöw daher ausdrücklich begrüßt.

Dennoch steckt auch hier der Teufel im Detail und längst nicht alles, was derzeit im politischen Alltagsgeschäft verwirklicht wird, entspricht dem, was im Sinne einer nachhaltigen Wirkung wünschenswert wäre. Es ist daher (...) an der Zeit, das Erreichte kritisch zu prüfen und dafür einzutreten, dass in der von Kurzfristigkeit und Pragmatismus geprägten Tagespolitik die großen Linien nicht verloren gehen. Dabei schlägt die vöw als Diskussionsgrundlage ein übergreifendes Prozesskriterium, nämlich die Austarierung des Spannungsfeldes von Vision und Pragmatismus, und vier inhaltliche Kriterien vor:

- Langfristigkeit

Ökologische Wirksamkeit

Soziale Ausgewogenheit und die

Psychologische Schlüsselwirkung. (...)

Auf dem bisher erfolgten ersten Schritt in Richtung einer zukunftsähigen Steuer- und Abgabenpolitik mit „ökologisch wahren Preisen“ darf sich weder die ökologische Wirtschaftsforschung noch die Politik ausruhen. Die Ökologische Steuerreform muss konsequent und glaubwürdig fortgeführt werden. Trotz der vorhandenen positiven Ansätze kritisiert die VöW die Ausgestaltung der ersten Stufe sowie besonders die (...) weiteren Stufen der Ökologischen Steuerreform als unsystematisch und nicht ausreichend. In den folgenden Punkten besteht erheblicher Reformbedarf.

\section{Systematik der Energiestevern}

Am 25. August hat die Bundesregierung den Gesetzentwurf mit vier weiteren Stufen der Ökologischen Steuerreform für die Jahre 2000 bis 2003 beschlossen, in denen die Energiesteuern auf Kraftstoffe jährlich um 6 Pf/Liter und auf Strom um $0,5 \mathrm{Pf} / \mathrm{kWh}$ ansteigen sollen. Im Gegenzug versprechen Finanz- und Arbeitsminister eine Senkung der Rentenversicherungsbeiträge um einen weiteren Beitragspunkt, jeweils paritätisch für Arbeitgeber und Arbeitnehmer.

Damit bleiben die Folgeschritte ab 2000 deutlich hinter dem 1999 getätigten ersten Schritt zurück. (...) Auch die ökologische Wirksamkeit [wird] weit hinter den gesteckten Erwartungen zurückbleiben. Hauptkritikpunkt ist nicht primär die Höhe der anvisierten Steuersätze für Strom und Kraftstoffe, sondern das Aussparen weiterer Steuererhöhungen bei Heizöl und Erdgas, die noch in der ersten Stufe mit in die Energiebesteuerung einbezogen waren, sowie bei Kohle und Kernbrennstoffen, die bisher nicht mit einer Energiesteuer belegt sind. (...) Die VöW fordert schon für die zweite Stufe eine Abkehr von der vorgesehenen selektiven Erhöhung der Steuersätze einzelner Energieträger. Die Bundesregierung sollte zu einer systematischen Energiebesteuerung übergehen. (...)

Darüber hinaus kann die Einführung einer Kohlendioxid-Komponente in der Energiebesteuerung mittelfristig sinnvoll und wünschenswert sein, um die Besteuerung der einzelnen Energieträger stärker an ihrer Klimaschädlichkeit ausrichten zu können.
Die VÖW begrüßt, dass die Bundesregierung mit ihrem Vorschlag für vier weitere Stufen der Ökologischen Steuerreform den Wahlzyklus durchbricht und so einen entscheidenden Schritt in Richtung Langfristigkeit des Reformprojektes Ökologische Steuerreform macht. Stetigkeit und Planbarkeit sind von zentraler Bedeutung und daher als wichtiger anzusehen als die exakte Höhe der in Zukunft festgelegten Energiesteuersätze. Allerdings wird diese Signalwirkung durch die Selektivität der weiteren Stufen beschädigt. Da zudem der Zeitraum bis 2003 nicht ausreichend ist, um die Energiepreise den „ökologisch wahren Preisen“ anzunähern und zumindest ansatzweise die externen Kosten zu internalisieren, wird die Bundesregierung aufgefordert, noch vor Verabschiedung der nächsten Stufen der Ökologischen Steuerreform im Herbst 1999 konkrete Aussagen über eine langfristige Fortführung der Ökologischen Steuerreform nach 2003 in allen ihren Komponenten zu machen. (...) Um umweltpolitisch wirksam sein zu können, sollten zumindest die Grundzüge der Energiebesteuerung bereits heute für die nächsten zehn bis 20 Jahre festgeschrieben werden. (...)

\section{Aufkommensneutralität und} Verwendung des Steueraufkommens

Die Koppelung [von Energiebesteuerung und Rentenbeitragssenkung] und die strikte Aufkommensneutralität wurden gewählt, um die Ökologische Steuerreform in Politik und Gesellschaft mehrheitsfähig zu machen, was zumindest für den Einstieg weitgehend gelungen ist. Dies ist aus ökologischer Sicht soweit nicht zu beanstanden, wie das umweltpolitische Ziel „Energieeinsparung und Reduktion der CO2-Emissionen" mindestens gleichberechtigt neben dem Ziel der Entlastung der Sozialversicherung rangiert. Umweltpolitisch nicht mehr hinnehmbar wäre allerdings eine Situation, in der die ökologische Vision verloren ginge und die Ökologische Steuerreform zu einer Art ,Sozialversicherungsfinanzierungssteuer" degradiert würde. (...)

Da ein abruptes Abrücken von der für die erste Stufe gewählten Kompensation der Energiesteuereinnahmen durch eine Senkung der Sozialversicherungsbeiträge der Akzeptanz der Ökologischen Steuerreform in der Bevölkerung und Wirtschaft eher schadet als nutzt, erscheint es aus pragmatischen Gründen akzeptabel, diesen Weg für die nächsten Stufen bis 2003 weiterzuverfolgen. (...) 
(c) 20I0 Authors; licensee IÖW and oekom verlag. This is an article distributed under the terms of the Creative Commons Attribution Non-Commercial No Derivates License (http://creativecommons.org/licenses/by-nc-nd/3.o/), which permits unrestricted use, distribution, and reproduction in any medium, provided the original work is properly cited. 